\title{
Identifying Heat Health Risks in the Urban Areas of Western Australia (WA) - An Enhanced Heat Vulnerability Assessment
} Qian (Chayn) Sun ${ }^{\mathrm{a},}$, Grace Yun ${ }^{\mathrm{b}}$, Ting Ling ${ }^{\mathrm{b}}$

${ }^{a}$ Geospatial Science, School of Science, RMIT University, chayn.sun@rmit.edu.au

${ }^{b}$ Department of Health, Western Australia,grace.yun@health.wa.gov.au ,ting.lin@gmail.com

* Corresponding author

Keywords: Urban Heat Island (UHI), heat vulnerability index (HVI), Geographically weighted regression (GWR)

\begin{abstract}
:
The impact of heat on health can be more significant in urban areas with more population and where the microclimate is often unintentionally modified to create the Urban Heat Island (UHI) effect. Extreme heat and UHI pose a risk to the health of vulnerable individuals, such as the elderly, the very young, and those need care. Vulnerability has become a central concept in climate change research and policy. To assess it, many studies have used equal weighted cumulative indices to aggregate multiple factors into a composite HVI (Heat Vulnerability Index) and analyse the differences and intensity across local areas and regions. However, the aggregation and equal weighting rationality, and the disregard of spatial correlation can result in inaccurate explanation on local vulnerabilities.

This study develops an enhanced index of population heat vulnerability (HVI) in Perth metropolitan area, Western Australia (WA), using environmental, demographic, and health-related risk factors for heat exposure, sensitivity and adaptive capability. Satellite derived urban heat island data and community profiles were integrated by a spatial risk assessment methodology to highlight potential heat health risk areas and build the foundations for mitigation and adaptation plans. Principal component analysis (PCA) was used to identify the key risk factors for heat vulnerability. Geographically weighted regression (GWR) were used to model the spatial relationships between temperature and other contributing factors to produce weights for calculating HVI. The index was finally mapped to produce a spatial representation of risk. The maps of spatial heat health vulnerability provide information to target heat-related health risks by aiding policy advisors, healthcare professionals, and ancillary services to develop heatwave preparedness plans at a local scale.
\end{abstract}

